\title{
The Finite Group Velocity of Quantum Spin Systems
}

\author{
Elliott H. Lieb* \\ Dept. of Mathematics, Massachusetts Institute of Technology \\ Cambridge, Massachusetts, USA \\ Derek W. Robinson ${ }^{\star \star}$ \\ Dept. of Physics, Univ. Aix-Marseille II, Marseille-Luminy, France
}

Received May 15, 1972

\begin{abstract}
It is shown that if $\Phi$ is a finite range interaction of a quantum spin system, $\tau_{t}^{\Phi}$ the associated group of time translations, $\tau_{x}$ the group of space translations, and $A, B$ local observables, then

$$
\lim _{\substack{|t| \rightarrow \infty \\|x|>v|t|}}\left\|\left[\tau_{t}^{\Phi} \tau_{x}(\mathrm{~A}), \mathrm{B}\right]\right\| \mathrm{e}^{\mu(v) t}=0
$$

whenever $v$ is sufficiently large $\left(v>V_{\Phi}\right)$ where $\mu(v)>0$. The physical content of the statement is that information can propagate in the system only with a finite group velocity.
\end{abstract}

\section{Introduction}

In [2] it was demonstrated that for a large class of translationally invariant interactions, time translations of quantum spin systems can be defined as automorphisms of a $C^{*}$-algebra, $\mathscr{A}$, of quasi-local observables, i.e. the abstract algebra generated by the spin operators. This should allow one to discuss features of the dynamical propagation of physical effects in an algebraic manner independent of the state of the system, i.e. independent of the kinematical data. It is expected that this propagation has many features in common with the propagation of waves in continuous matter and the point of this paper is to demonstrate such a feature, namely a finite bound for the group velocity of a system with finite range interaction. This result is obtained by a simple estimation derived from the equations of motion and it is possible that more detailed estimations would give more precise information of the form of spin-wave propagation. We briefly discuss this possibility at the end of Section 3.

$\star$ Work supported by National Science Foundation Grant N ${ }^{\circ}$ : GP-31674X.

$\star \star$ Work supported by National Science Foundation Grants $N^{\circ}$ : GP-31239 X and GP-30819X. 


\section{Basic Notation}

We use the formalism introduced in [1] and [2]. For completeness we recall the basic definitions which will be used in the sequel.

The kinematics of a quantum spin system constrained to a $v$-dimensional cubic lattice, $\mathbb{Z}^{v}$, are introduced by associating with each point $x \in \mathbb{Z}^{v}$ an $N$-dimensional vector space $\mathscr{H}_{x}$ and with each finite set $\Lambda \subset \mathbb{Z}^{v}$ the direct product space

$$
\mathscr{H}_{\Lambda}=\prod_{x \in \Lambda}^{\otimes} \mathscr{H}_{x} .
$$

The algebra of strictly local observables, $\mathscr{A}_{A}$, of the subsystem $\Lambda$, is defined to be the algebra of all matrices acting on $\mathscr{H}_{\Lambda}$. If $\Lambda_{1} \subset \Lambda_{2}$, the algebra $\mathscr{A}_{\Lambda_{1}}$ acting on $\mathscr{H}_{\Lambda_{1}}$ can be identified with the algebra $\mathscr{A}_{\Lambda_{1}} \otimes \mathbb{1}_{\Lambda_{2} \backslash \Lambda_{1}}$ acting on $\mathscr{H}_{\Lambda_{2}}\left(\mathbb{1}_{\Lambda_{2} \backslash \Lambda_{1}}\right.$ is the identity operator on $\left.\mathscr{H}_{\Lambda_{2} \backslash \Lambda_{1}}\right)$ and with this identification $\mathscr{A}_{\Lambda_{1}} \subset \mathscr{A}_{A_{2}}$. Due to this isotony relation the set theoretic union of all $\mathscr{A}_{\Lambda}$, with $\Lambda \subset \mathbb{Z}^{v}$ finite is a normed ${ }^{*}$-algebra and we define the completion of this algebra to be the $C^{*}$-algebra $\mathscr{A}$ of (quasi-)local kinematical observables of the spin system. The group $\mathbb{Z}^{v}$ of space translations is a subgroup of the automorphism group of $\mathscr{A}$, and we denote the action of this group by $A \in \mathscr{A}_{A} \rightarrow \tau_{x} A \in \mathscr{A}_{A+x}$ for $x \in \mathbb{Z}^{v}$.

To define the dynamics of our system we introduce an interaction $\Phi$ as a function from the finite sets $X \subset \mathbb{Z}^{v}$ to elements $\Phi(X) \subset \mathscr{A}_{X}$. In contrast to [2] we will only consider finite range interactions in the sequel. Thus we demand that $\Phi$ satisfies:

1. $\Phi(X)$ is Hermitian for $X \subset \mathbb{Z}^{v}$.

2. $\Phi(X+a)=\tau_{a} \Phi(X)$ for $X \subset \mathbb{Z}^{v}$ and $a \in \mathbb{Z}^{v}$.

3. The union $R_{\Phi}$ of all $X$ such that $X \ni 0$ and $\Phi(X) \neq 0$ is a finite subset of $\mathbb{Z}^{\nu}$.

[Physically only particles situated at the points $x \in R_{\Phi}$ have a nonzero sinteraction with a particle at the origin.]

The Hamiltonian of a finite system $\Lambda$ with interaction $\Phi$ is defined by:

$$
H_{\Phi}(\Lambda)=\sum_{X \subset A} \Phi(X) .
$$

In [2] it was established that each interaction $\Phi$ defines a strongly continuous, one-parameter group of automorphisms $\tau_{t}^{\Phi}$ of $\mathscr{A}$. Explicitly we have for each $A \in \mathscr{A}$ and $t \in R$ an element $\tau_{t}^{\Phi}(A) \in \mathscr{A}$ such that:

$$
\begin{gathered}
\lim _{A \rightarrow \infty}\left\|\tau_{t}^{\Phi}(A)-e^{i t H_{\Phi}(A)} A e^{-i t H_{\Phi}(A)}\right\|=0 \\
\lim _{t \rightarrow 0}\left\|\tau_{t}^{\Phi}(A)-A\right\|=0 \\
\tau_{t}^{\Phi}(A B)=\tau_{t}^{\Phi}(A) \tau_{t}^{\Phi}(B), \quad \text { etc. } \ldots
\end{gathered}
$$


In fact $t \rightarrow \tau_{t}^{\Phi}(A)$ with $A \in \mathscr{A}_{A}$ is analytic in a strip $|\operatorname{Imt}|<a_{\Phi}$ with $a_{\Phi}>0$ and further:

$$
\lim _{\Lambda \rightarrow \infty}\left\|\frac{d}{d t} \tau_{t}^{\Phi}(A)-\frac{d}{d t} e^{i t H_{\Phi}(\Lambda)} A e^{-i t H_{\Phi}(A)}\right\|=0 .
$$

For details see [2].

\section{Local Commutativity}

Our aim is to discuss the behaviour of commutators

$$
C_{A, B}(x, t)=\left[\tau_{t}^{\Phi} \tau_{x}(A), B\right]
$$

for $A$ and $B$ strictly local, i.e. contained in some $\mathscr{A}_{A}$. We wish to examine the magnitude of these commutators for large $x$ and $t$ and to show that information propagates with a finite group velocity $V_{\Phi}$. More precisely, we have the following.

Theorem. For each finite range interaction $\Phi$ there exists a finite group velocity $V_{\Phi}$ and a strictly positive increasing function $\mu$ such that for $v>V_{\Phi}$

$$
\lim _{\substack{|t| \rightarrow \infty \\|x|>v|t|}} e^{\mu(v)|t|}\left\|\left[\tau_{t}^{\Phi} \tau_{x}(A), B\right]\right\|=0
$$

for all strictly local $A$ and $B$.

Proof. First we note that it is sufficient to prove the theorem for $A, B \in \mathscr{A}_{\{0\}}$. This is because each strictly local $A, B \in \mathscr{A}_{A}$ can be written as a polynomial in elements of $\mathscr{A}_{\{x\}}$ with $x \in \Lambda$. Hence the norm of the general commutator can be bounded above by a finite sum of norms of similar commutators but with $A \in \mathscr{A}_{\{x\}}, B \in \mathscr{A}_{\{y\}}$ and $x, y \in \Lambda$. Using translation invariance each of these commutators can be reduced to a commutator with $A, B \in \mathscr{A}_{\{0\}}$.

As $\mathscr{H}_{0}$ is finite dimensional we can choose a finite basis $a_{1}, \ldots, a_{N^{2}}$ of $\mathscr{A}_{\{0\}}$ closed under multiplication with $\left\|a_{i}\right\|=1$ and such that every $A \in \mathscr{A}_{\{0\}}$ has a unique decomposition of the form

$$
A=\sum_{i=1}^{N^{2}} C_{i}(A) a_{i}, \quad C_{i}(A) \in \mathbb{C} .
$$

Further, if $A \in \mathscr{A}_{\left\{x_{1}, \ldots, x n\right\}}$, then $A$ has a unique decomposition as a polynomial

$$
A=\sum_{i_{1}=1}^{N^{2}} \ldots \sum_{i n=1}^{N^{2}} e\left(i_{1}, \ldots, i_{n} ; A\right) \prod_{j=1}^{n} \tau_{x_{j}}\left(a_{i_{j}}\right), \quad e \in \mathbb{C} .
$$


Next, with $B \in \mathscr{A}_{\{0\}}$ fixed, consider

and

$$
C_{i}(x, t)=\left[\tau_{t}^{\Phi} \tau_{x}\left(a_{i}\right), B\right]
$$

$$
F_{i}(x, t)=\left\|C_{i}(x, t)\right\| \text {. }
$$

From the definition of the time translation automorphisms and their properties cited in the previous section, one obtains the differential equations

$$
\frac{d}{d t} C_{i}(x, t)=i \sum_{X \ni 0}\left[\tau_{t}^{\Phi} \tau_{x}\left(\left[\Phi(X), a_{i}\right]\right), B\right] .
$$

For each $X$ in this sum, the corresponding $\Phi(X)$ can be written as a polynomial in the set of elements $\tau_{y}\left(a_{j}\right), y \in X, j=1,2, \ldots, N^{2}$. The commutator $D_{i}(X)=\left[\Phi(X), a_{i}\right]$ is then a polynomial of the same kind. Each monomial, $M$, in $D_{i}(X)$ is of the form

Hence

$$
M=\prod_{y \in X} \tau_{y}\left(a_{j(y)}\right) .
$$

$$
\tau_{t}^{\Phi} \tau_{x}(M)=\prod_{y \in X} \tau_{t}^{\Phi} \tau_{x+y}\left(a_{j(y)}\right) .
$$

The commutator $\left[\tau_{t}^{\Phi} \tau_{x}(M), B\right]$ will have $N(X)$ terms (the number of points in $X$ ), each obtained by taking the commutator $\left[\tau_{t}^{\Phi} \tau_{x+y}\left(a_{i(y)}\right), B\right]$ and leaving the other elements in $\tau_{t}^{\Phi} \tau_{x}(M)$ as coefficients. Each of these coefficients has norm one (the $a_{j}$ have norm one and automorphisms preserve the norm). Hence

and

$$
\left\|\left[\tau_{t}^{\Phi} \tau_{x}(M), B\right]\right\| \leqq \sum_{y \in X}\left\|\left[\tau_{t}^{\Phi} \tau_{x+y}\left(a_{j(y)}\right), B\right]\right\|
$$

$$
\begin{aligned}
\left\|\frac{d C_{i}(x, t)}{d t}\right\| & \leqq \sum_{y \in R^{\Phi}} \sum_{j=1}^{N^{2}} d_{j i}(\Phi ; y) F_{j}(x+y, t) \\
& \leqq \sum_{y \in R^{\Phi}} \sum_{j=1}^{N^{2}} d_{j i}(\Phi) F_{j}(x+y, t) \\
& =(\mathscr{L} F)_{i}(x, t)
\end{aligned}
$$

where $d_{j i}(\Phi ; y)$ is a non-negative coefficient that depends on the interaction $\Phi$ and the point $y$, whilst $d_{j i}(\Phi)=\max _{y \in R_{\Phi}} d_{j i}(\Phi ; y)$. Using the triangle inequality it is easy to verify that

$$
\left\|\frac{d C_{i}(x, t)}{d t}\right\| \geqq \mathscr{D}_{t} F_{i}(x, t),
$$

where $\mathscr{D}_{t}$ denotes the upper derivative, i.e.

$$
\left(\mathscr{D}_{t} f\right)(t)=\lim \sup _{\varepsilon \rightarrow 0} \frac{f(t+\varepsilon)-f(t)}{\varepsilon} .
$$


Thus $F_{i}$ satisfies the differential inequality

$$
\left(\mathscr{D}_{t} F\right)_{i}(x, t) \leqq(\mathscr{L} F)_{i}(x, t)
$$

and the initial data

where

$$
\begin{gathered}
F_{i}(x, 0)=\delta_{x, 0} \omega_{i} \\
\omega_{i}=\left\|\left[a_{i}, B\right]\right\| .
\end{gathered}
$$

[For the sequel note that we could have used the analyticity of $\tau_{t}^{\Phi}$, etc. to deduce the partial difference inequality

$$
\frac{F_{i}(x, t+h)-F_{i}(x, t)}{h} \leqq(\mathscr{L} F)_{i}(x, t)+h K
$$

for $h$ small, where $K$ is a positive constant. This would have avoided the introduction of the upper derivative to circumvent the possible nondifferentiability of $F_{i}$.]

Next, consider the Green's function $G_{i}^{k}(x, t)$ defined by the differential equations

and

$$
\frac{\partial G_{i}^{k}(x, t)}{\partial t}=\left(\overline{\mathscr{L}} G^{k}\right)_{i}(x, t)
$$

$$
G_{j}^{k}(x, 0)=\delta_{x, 0} \delta_{j, k}
$$

$(\overline{\mathscr{L}}$ is the adjoint of $\mathscr{L})$.

Subsequently we shall use the non-negativity of the coefficients $d_{i j}(\Phi)$ to conclude that the $G_{i}^{k}$ are non-negative, for all $t>0, x, i$ and $k$, that they are real analytic in $t$, and that there exists a constant $V_{\Phi}>0$, and an increasing function $\mu(v)>0$ such that

$$
\lim _{\substack{t \rightarrow \infty \\|x|>v t}} e^{\mu(v) t} G_{i}^{k}(x, t)=0, \quad v>V_{\Phi} .
$$

For the moment we accept these properties and reconsider the differential inequalities satisfied by the $F_{i}$. We deduce from the nonnegativity of the $G_{i}^{k}$ that

$$
\begin{aligned}
\sum_{i=1}^{N^{2}} & \sum_{x \in \mathbb{Z}} \int_{0}^{t^{\prime}} d t G_{i}^{k}\left(y-x, t^{\prime}-t\right)\left(\mathscr{D}_{t} F\right)_{i}(x, t) \\
& \leqq \sum_{i=1}^{N^{2}} \sum_{x \in \mathbb{Z}} \int_{0}^{t^{\prime}} d t G_{i}^{k}\left(y-x, t^{\prime}-t\right)(\mathscr{L} F)_{i}(x, t) .
\end{aligned}
$$

Upon integrating by parts on the left hand side, a process which is legitimized by the real analyticity of $G_{i}^{k}$ in $t$ and the interpretation of the differential inequality as a partial difference inequality, we find that

$$
0 \leqq F_{k}(x, t) \leqq \sum_{i} G_{i}^{k}(x, t) \omega_{i}
$$


which concludes the proof for $t \rightarrow \infty$. The limit $t \rightarrow-\infty$ is easily handled by noting that $\tau_{-t}^{\Phi}=\tau_{t}^{-\Phi}$ and the differential inequality that we have derived is invariant under interchange of $\Phi$ by $-\Phi$.

The task remains of corroborating our assertions concerning the Greens functions. These functions can be explicitly calculated in matrix form and one finds:

$$
\boldsymbol{G}(x, t)=\frac{1}{(2 \pi)^{v}} \int_{0}^{2 \pi} d \theta_{1} \ldots \int_{0}^{2 \pi} d \theta_{v} e^{i \theta \cdot x} \exp \{t \omega(\theta) \boldsymbol{D}\}
$$

where $\boldsymbol{D}$ and $\boldsymbol{G}$ are matrices whose $(i, j)$ elements are respectively $d_{i j}$ and $\boldsymbol{G}_{j}^{i}(x, t)$ and

$$
\omega(\theta)=\sum_{y \in R_{\Phi}} e^{+i \theta \cdot y} .
$$

The analyticity of $G$ follows from this formula. The non-negativity is best seen from the differential equation which gives directly

$$
G_{i}^{k}(x, 0) \geqq 0, \frac{d G_{i}^{k}}{d t}(x, 0) \geqq 0, \ldots, \frac{d^{n} G_{i}^{k}}{d t^{n}}(x, 0) \geqq 0,
$$

Next, note that for all $y \in R^{v}$

$$
|\omega(\theta+i \gamma)| \leqq \omega(i \gamma)=\sum_{y \in R_{\Phi}} e^{-\gamma \cdot y},
$$

and that $\omega$ has period $2 \pi$ in each of the coordinates $\theta_{i}$. Thus by contour integration we have

and

$$
\boldsymbol{G}(v t, t)=\frac{1}{(2 \pi)^{v}} \int_{0}^{2 \pi} d \theta_{1} \ldots \int_{0}^{2 \pi} d \theta_{v} e^{i(\theta+i \gamma) \cdot v t} \exp \{t \omega(\theta+i \gamma) \boldsymbol{D}\}
$$

$$
\|\boldsymbol{G}(v t, t)\| \leqq e^{-\gamma \cdot v t} \exp \{t \omega(i \gamma)\|\boldsymbol{D}\|\} .
$$

If $|v|$ is sufficiently large there is certainly a $\gamma$ such that

$$
\gamma \cdot v \geqq\|\boldsymbol{D}\| \omega(i \gamma)
$$

and the asymptotic properties of $G$ follow immediately.

Remark 1. The conclusions of the above theorem can be established, by a simple refinement of the above argument, for a class of infinite range interactions with exponential decrease. An example of such an interaction would be a two-body interaction (i.e. $\Phi(X)=0$ unless $N(X)=2$ ) with the property that

$$
\|\Phi(\{0, x\})\| \leqq e^{-a|x|}, \quad a>0 .
$$

It is also clear that the argument can be refined to apply to interactions which are more slowly decreasing, but in this case the commutators will not tend to zero exponentially. Instead their decrease will be linked to that of the interaction. 
Remark 2. Our method of estimating the group velocity $V_{\Phi}$ is crude, but nevertheless yields an upper bound that is not difficult to calculate in specific cases, e.g. the nearest neighbour Heisenberg model. One could systematically improve our method of calculating $V_{\Phi}$. To do so one would prescribe a hierarchy of commutators; the first category would include all two point commutators, the second all three point commutators, etc. In this way one obtains a set of coupled diffusion equations with the property that truncation at any stage, and estimation of the above type, gives an upper bound for $V_{\Phi}$. It is interesting to ask whether these successive approximations actually converge to the true $V_{\Phi}$.

Finally for each finite range interaction $\Phi, \mathscr{A}$ is asymptotically abelian for the group of space-time automorphisms $(x, t) \in \mathbb{Z}^{v} x R \rightarrow \tau_{x} \tau_{t}^{\Phi}$ in the cone $C_{\Phi}=\left\{(x, t) ;|x|>V_{\Phi}|t|\right\}$, i.e.

$$
\lim _{\substack{|t| \rightarrow \infty \\|x|>V_{\Phi}|t|}}\left\|\left[\tau_{x} \tau_{t}^{\Phi}(A), B\right]\right\|=0
$$

for all $A, B \in \mathscr{A}$. This follows because the $A$ and $B$ can be approximated in norm by strictly local elements $A_{L}$ and $B_{L}$ of $\mathscr{A}$ and further

$$
\left\|\tau_{x} \tau_{t}^{\Phi}\left(A-A_{L}\right)\right\|=\left\|A-A_{L}\right\|, \quad \text { etc. }
$$

for all $(x, t) \in \mathbb{Z}^{v} x R$.

Acknowledgments. This work was undertaken whilst one of us (D. W. Robinson) was a guest of the Department of Physics, Harvard University. The hospitality of Profs. S. Coleman, S. Glashow and A. Jaffe during this period is gratefully acknowledged.

\title{
References
}

1. Robinson, D. W.: Commun. math. Phys. 6, 151 (1967).

2. Robinson, D. W.: Commun. math. Phys. 7, 337 (1968). - See also; Streater, R.F.: Commun. math. Phys. 7, 93 (1968). - Ruskai, M. B.: Commun. math. Phys. 20, 193 (1971).

\author{
D. W. Robinson \\ Centre de Physique Théorique \\ C.N.R.S. \\ 31, chemin J. Aiguier \\ F-13 Marseille $9^{\circ}$, France
}


